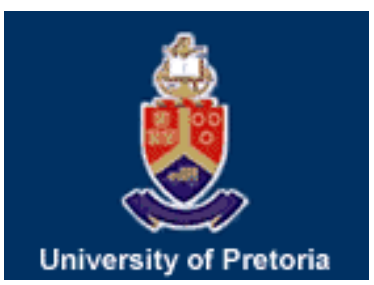

University of Pretoria

Department of Economics Working Paper Series

\author{
Subjective Life Expectancy \\ Nicky Nicholls \\ University of Pretoria \\ Alexander Zimper \\ University of Pretoria \\ Working Paper: 2014-10 \\ March 2014
}

Department of Economics

University of Pretoria

0002, Pretoria

South Africa

Tel: +27124202413 


\section{Subjective Life Expectancy}

Nicky Nicholls, University of Pretoria, South Africa, email: nicky.nicholls@gmail.com

Alexander Zimper, University of Pretoria, South Africa, email: alexander.zimper@up.ac.za

ABSTRACT. Individuals' subjective life-expectancy, as elicited in large-scale surveys, shows underestimation of survival chances at young versus overestimation at old ages. These distorted perceptions of objective survival chances may cause young people to save too little and old people to accumulate too much wealth late in life with respect to the rational expectations benchmark model. Alternative explanations for these differences between perceived and objective survival chances include cognitive shortcomings or/and preference-based (motivational) reasons. To know the exact nature of these differences would be relevant for judging policy interventions that aim at influencing people's savings behaviour.

\section{Introduction}

Subjective Life Expectancy (SLE) refers to individuals' expectations of their likelihood of living up to a given age. Such expectations are relevant to important economic decisions of households such as consumption versus savings behaviour, the purchase of life insurance and annuities, choice of retirement age, fertility decisions and investment in child welfare and education. Also risky health behaviours such as smoking and drug use are influenced by SLE because people may continue/stop such behaviours if they underestimate/overestimate reductions in their life-expectancy resulting from these behaviours.

In line with the 'rational expectations' paradigm, social scientists have traditionally modelled subjective life expectations as (projected) objective survival probabilities. In recent years, however, the data from large-scale surveys has shown that projected objective life expectancy is not a good surrogate for SLE. In particular, there is strong evidence that young people underestimate whereas old people overestimate their chances to survive into the future. This pattern of differences between SLE and objective survival chances might contribute towards explanations for empirically observed savings puzzles such as 'undersaving at younger' versus 'oversaving at older ages' in the sense that while younger individuals do not adequately save for retirement, older individuals retain too much wealth. 
At this point, there is no common agreement in the literature about the exact reasons for these differences between SLE and objective survival chances. The two main candidates discussed in the decision-theoretic literature are 'cognitive shortcomings', on the one hand, and preference-based 'motivational reasons', on the other hand. Depending on which alternative explanation is deemed the more appropriate, there are different recommendations for policy measures as well as different justifications for policy interventions such as, e.g., mandatory savings programs or pension systems; the design of annuities and life insurance products; policies guiding individual decisions about retirement savings and what to invest in.

Suppose, for instance, that the differences between SLE and objective survival chances are caused by cognitive shortcomings in the sense that individuals do not correctly form and update probabilities. In this case, policy measures that emphasize better probabilistic education or better information on survival chances or both may be appropriate because they assist people in avoiding undesired errors and mistakes.

Alternatively, suppose that the differences result from individuals' preferences for decision making under uncertainty in the sense that the reported SLE stand for individuals' decision weights that do not coincide with objective probabilities. In that case, policy measures that thwart the phenomena of undersaving at young and oversaving at old ages would have a paternalistic character in that they interfere with individuals' preferences.

In the remainder of this article we briefly review existing surveys and their methods for eliciting SLE. This is followed by a discussion of the main findings of these surveys, in the form of differences between SLE and objective survival chances, as well as a brief overview of different "explanations" for these differences.

\section{Eliciting SLE in surveys}

Subjective life expectancies are elicited in the form of subjective survival beliefs, that is, the probability of surviving to a specified age. Such probabilistic data on SLE is, for instance, collected in the US Health and Retirement Survey (HRS), the English Longitudinal Study of Ageing (ELSA), the Survey of Health Ageing and Retirement in Europe (SHARE) and the Korean Longitudinal Study of Ageing. All these surveys use very similar structures for SLE questions. Since the HRS has been most widely used in research, we discuss its question structure and findings in some more detail below. The University of Michigan Health and Retirement Study (HRS) was launched in 1992 with a cohort of individuals ages 51-61 and their spouses. In 1998, the sample was updated to be representative of all age groups aged above 50 . The HRS is a longitudinal panel study surveying a representative 
sample of Americans over the age of 50 every two years (sample size is now over 26,000 ) with a new six-year cohort of 51-56 year olds and spouses now being added every six years (2004 and 2010). The long-running nature of this survey means that outcomes can now be measured and compared to expectations.

In the HRS, the SLE question is asked as follows:

What is the percent chance that you will live to be 75 or more?

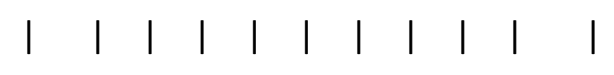

00102030405060708090100

where: $00=$ Absolutely no chance and $100=$ Absolutely certain

The target age (75 in the example above) varies based on the current age of the respondent, with target ages as follows in use since 2006 (some of the younger respondents are asked about a target age of 75 in addition to the question below):

Table 1: HRS target ages

\begin{tabular}{lc}
\hline Age at Interview & Target Age \\
\hline $50-64$ & 85 \\
$65-69$ & 80 \\
$70-74$ & 85 \\
$75-79$ & 90 \\
$80-85$ & 95 \\
$85-89$ & 100 \\
\hline
\end{tabular}

One criticism of the above probabilistic approach is that individuals might not have an accurate understanding of probabilities and might therefore report incorrect probabilities. To address this criticism, HRS respondents are helped---prior to asking the life expectancy question---with probabilistic thinking through a training question asking, e.g., about the probability of rain tomorrow. Moreover, Hurd (2009) argues that although these probabilities might be 'incorrect', they should nonetheless represent the decision weights used by individuals when making decisions.

Another concern about the elicitation of SLE in the aforementioned surveys is the question of whether respondents honestly reveal their expectations in the absence of any incentives for honest responses. Manski (2004) argues against the relevance of this concern because the responses turn out to be sensible and internally consistent. For example, SLE co-vary in the HRS with known risk factors (e.g., smoking and drinking) in the expected way based on objective data. Similarly, SLE were found to increase with income, wealth and schooling as expected (Hurd \& McGarry, 2002). 


\section{Differences between SLE and objective survival chances}

Objective survival probabilities are based on life/mortality tables. These compile data of actual recent lifespans in the population in order to predict life expectancy for people of different ages. Cohort life tables directly include cohort-effects as mortality rates can be looked at based on year of birth (cohort data on mortality in the US and various other countries are available in the Human Mortality Database). In attempting to better predict life expectancy particularly for younger cohorts in the population (where medical advances might render historical data less useful in estimating life expectancy), models have been developed to account for the change over time of mortality rates, with that of Lee and Carter (1992) being most prominent.

The HRS SLE show systematic differences from objective survival chance: As respondents get older, they become more optimistic about their likelihood to survive to a given age. Respondents are pessimistic (relative to life table predictions) until age 69, realistic from age 70 to 74 and optimistic at age 75 and over. Figure 1 (taken from Groneck, Ludwig \& Zimper, 2014) illustrates these differences for female and male respondents, respectively. Both graphs show deviations in percentage points of SLE from objective survival rates based on cohort life table data. Future objective data is predicted with the Lee-Carter procedure. Each bar depicts the difference of unconditional survival probabilities to a specific target age that varies with the age of the interview according to Table 1. 

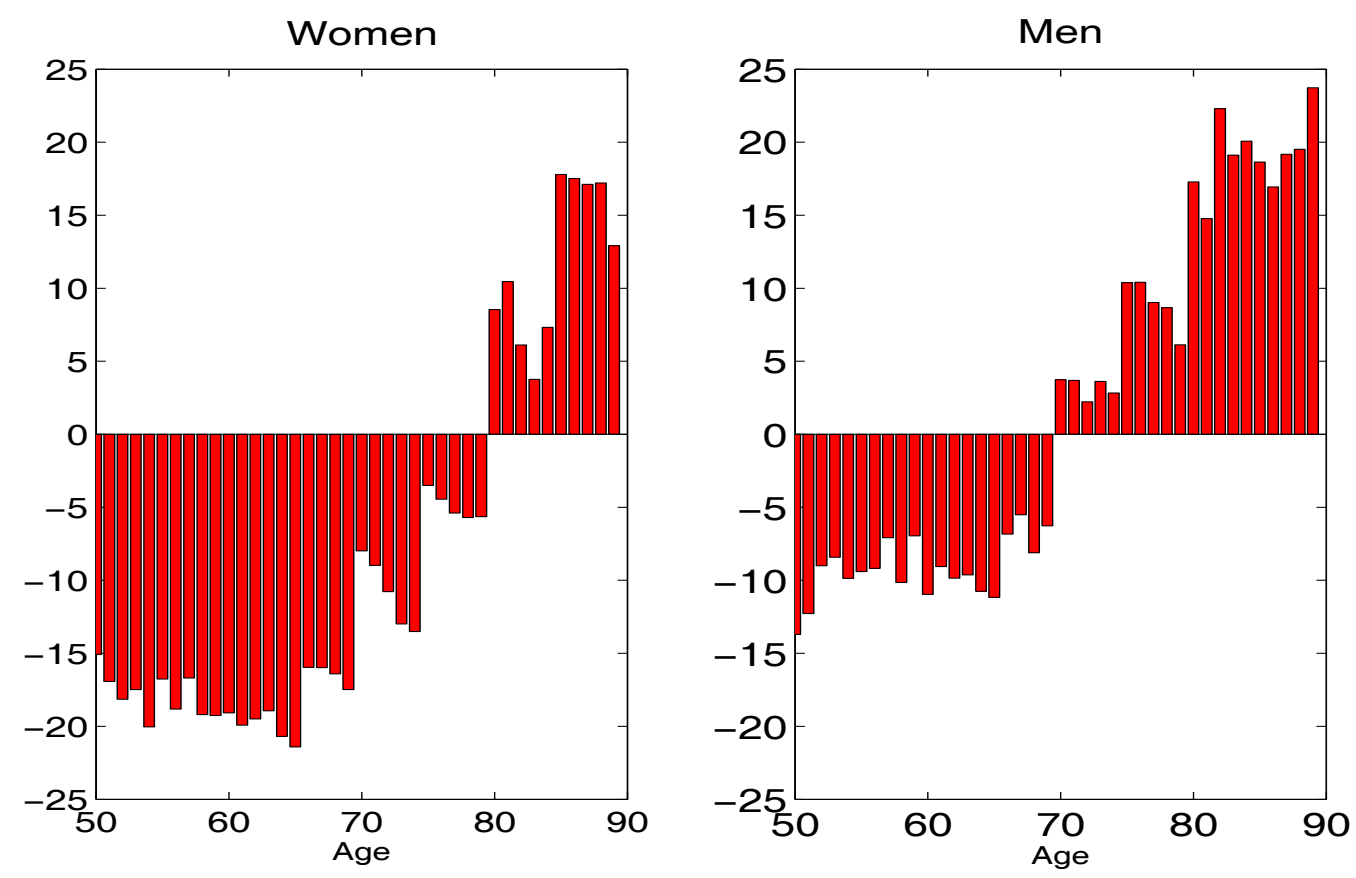

Certain systematic differences between SLE and projected life table data are to be expected based on sampling effects. Hurd and McGarry (2002) point to two reasons for higher life expectancy in the sampled group with reference to the HRS sample. This sample excludes institutionalised adults (who would be expected to have higher than average mortality rates); and also does not elicit subjective survival expectations where responses are given by proxy (respondents interviewed by proxy are also likely to have lower-than-average survival rates).

However, underestimation at younger versus overestimation at older ages are persistent effects whose magnitude cannot be explained by sampling effects alone. These effects were first noted with a non-representative sample by Hamermesh (1985) and have subsequently been observed in HRS data (Gan, Hurd \& McFadden, 2005; Hurd \& McGarry, 1995) as well as in SHARE data (Peracchi \& Perotti, 2012).

\section{4. "Explaining" the differences}

Several possible explanations for the observed differences between SLE and objective survival chances have been put forward in the literature. Kastenbaum (2000) offers the psychological ad hoc explanation that people prefer to minimize their encounters with death. As people age, they might therefore rely increasingly on their optimistic assessments of survival in order to avoid such cognitive encounters. Another ad hoc explanation in terms of cognitive shortcomings is that the true 
probability of survival to increasing ages declines rapidly to the effect that individuals' survival expectations might simply not keep up with this decline (Kezdi \& Willis, 2013).

Beyond these psychological ad hoc explanations, more formal decision theoretic modes have been developed that can generate the observed differences. For example, experiments which assume that individuals have preferences in line with Cumulative Prospect Theory (Tversky \& Kahneman, 1992) (CPT) typically elicit inverse-S shaped probability weighting functions. Such inverse-S weighting functions imply that individuals overweight small probabilities whereas they underweight large probabilities. If the SLE represent such weighted probabilities (i.e., decision weights), underestimation (overestimation) of survival probabilities by younger (older) people would occur whenever these people are typical CPT decision makers because survival probabilities are larger for younger than for older people.

Within such a CPT framework, Wu, Stevens and Thorp (2013) consider data from the Australian Retirement Plans and Retirement Incomes survey (2011) where respondents are asked to provide subjective survival probabilities for 10 different target ages. Based on the responses to these questions, these authors estimate a life cycle model where mortality beliefs are updated in line with changes in optimism/pessimism over the life cycle.

Ludwig and Zimper (2013) develop a dynamic CPT model of SLE that is based on a non-standard Bayesian learning model. Their point of departure is that under standard Bayesian learning SLE would converge over the individuals' life-time towards objective survival chances because the amount of information increases as time passes and the individual learns more. In contrast to this consistency result for Bayesian estimates, the non-standard Bayesian learning model in Ludwig and Zimper (2013) allows for diverging SLE when the individuals receive more information. In particular, these authors' learning model generates the observed underestimation at younger ages and overestimation at older ages for standard values of CPT probability weighting parameters. Groneck, Ludwig and Zimper (2014) merge the dynamic CPT model of Ludwig and Zimper (2013) with a life-cycle model to obtain undersaving at young versus oversaving at old ages. Although this model can thus capture the qualitative effects observed in empirical studies on savings behaviour, the authors also argue that dynamic CPT decision making alone cannot fully explain the magnitude of these effects.

The aforementioned CPT models leave it open whether the differences between SLE and objective survival chances are caused by cognitive failures or rather by preference-based motivational reasons. Originally, the probability weighting in CPT had been developed to represent preferences under risk/uncertainty that could accommodate systemic violations of expected utility in the form of Allais/Ellsberg paradoxes. According to this preference-based approach, the pattern of Figure 1 
could be explained by relative pessimism (under ambiguity) at young and relative optimism (under ambiguity) at old ages. Such a preference-based explanation would be in line with Kastenbaum's (2000) psychological explanation that older people minimize their cognitive encounters with death. Giving older people incentives to decumulate wealth in line with their objectively low survival chances would then be a bad policy measure with respect to these people's preferences. Alternatively, Peter Wakker (2010) argues that probability weighting under CPT may reflect cognitive failures in the form of likelihood insensitivity. Likelihood insensitivity describes the situation that people do not sufficiently sharply distinguish between non-degenerate probabilities such as in "fifty-fifty" probability judgements.

Finally, let us mention that a recent experimental study by Abdellaoui, Baillon, Placido, and Wakker (2011) indicates that CPT probability weights result from a combination of likelihood insensitivity and preferences whereby preferences play a more dominant role when there is more ambiguity with respect to the probability generating source. If it was the case that these findings also apply to SLE as expressed CPT decision weights, then the differences between SLE and objective survival chances would result from a combination of cognitive shortcomings and individuals' preferences under uncertainty.

\section{REFERENCES}

Abdellaoui, M., Baillon, A., Placido, L., \& Wakker, P.P. (2011). The rich domain of uncertainty: Source functions and their experimental implementation. American Economic Review, 101(2), 695-723.

Gan, L., Hurd, M., \& McFadden, D. (2005). Individual subjective survival curves. In D. Wide (Ed.), Analyses in the economics of aging. Cambridge: NBER Books, National Bureau of Economic Research.

Groneck, M., Ludwig, A., \& Zimper, A. (2014). A life-cycle model with ambiguous survival beliefs. Working Paper. Center for Macroeconomic Research (University of Cologne) and University of Pretoria.

Hamermesh, D. S. (1985). Expectations, life expectancy, and economic behavior. The Quarterly Journal of Economics, 100(2), 389-408.

Hurd, M. D., \& McGarry, K. (1995). Evaluation of the subjective probabilities of survival in the health and retirement study. The Journal of Human Resources, 30, 268-292. 
Hurd, M., \& McGarry, K. (2002). The predictive validity of subjective probabilities of survival. The Economic Journal, 112, 966-985.

Hurd, M. (2009). Subjective probabilities in household surveys. Annual Review of Economics, 2009(1), 543-562.

Kahneman, D., \& Tversky, A. (1979). Prospect theory: An analysis of decision under risk. Econometrica, 47(2), 263-292.

Kastenbaum, R. (2000). The psychology of death. New York: Springer Publishing Company.

Kezdi, G., \& Willis, R. (2013). Expectations, aging and cognitive decline. Working Paper. Central European University, IE-CERSHAS, University of Michigan and NBER.

Lee, R., \& Carter, L. (1992). Modeling and forecasting U.S. mortality. Journal of the American Statistical Association, 87(419), 659-671.

Ludwig, A., \& Zimper, A. (2013). A parsimonious model of subjective life expectancy. Theory and Decision, 75, 519-542.

Manski, C.F. (2004). Measuring expectations. Econometrica, 72(5), 1329-1376.

Peracchi, P., \& Perotti, V. (2012). Subjective survival probabilities and life tables: Evidence from Europe. Working Paper. Tor Vergata University, EIEF and the World Bank.

Tversky, A., \& Kahneman, D. (1992). Advances in prospect theory: cumulative representations of uncertainty. Journal of Risk and Uncertainty, 5(4), 297-323.

Wakker, P. P. (2010). Prospect theory for risk and ambiguity. Cambridge: Cambridge University Press.

Wu, S., Stevens, R., \& Thorp, S. (2013). Die young or live long: Modeling subjective survival probabilities. Working Paper. Centre for Study of Choice (CenSoc).

\section{FURTHER READING}

Delavande, A., \& Rohwedder, S. (2008). Differential mortality in Europe and the U.S. Estimates based on subjective probabilities of survival. Working Paper. RAND: WR-613. 
Elder, T. (2013). The predictive validity of subjective mortality expectations: Evidence from the health and retirement study. Demography, 50, 569-589.

\section{BIOS}

Nicky Nicholls received a Masters in Economics from the University of the Witwatersrand, Johannesburg. Currently, she is enrolled in the PhD programme of the Economics Department of the University of Pretoria. Nicky's PhD research focuses on the link between the Economics of Aging and descriptive Decision Theory.

Alex Zimper is a Professor at the Economics Department of the University of Pretoria. In 2004 he received his PhD in Economics from the University of Mannheim, Germany, for his dissertation entitled "Contributions to the Theory of Solution Concepts for Strategic Games". Since 2006 he has been teaching Economic Theory and Financial Economics at leading South African Universities such as UCT, UJ, and WITS. Besides the Economics of Aging, his research interests include Decision Theory, Asset Pricing, Banking Regulation, Bayesian Learning under Ambiguity, and Bounded Rationality.

KEYWORDS: Health Retirement Study, Undersaving, Oversaving, Cumulative Prospect Theory, Likelihood Insensitivity 